\title{
Positive Surgical Margins Predict Progression-free Survival After Nephron-sparing Surgery for Renal Cell Carcinoma: Results From a Single Center Cohort of 459 Cases With a Minimum Follow-up of 5 Years
}

\author{
Riccardo Tellini, ${ }^{1}$ Alessandro Antonelli, ${ }^{1}$ Regina Tardanico, ${ }^{2}$ Simona Fisogni, ${ }^{2}$ \\ Alessandro Veccia, ${ }^{1}$ Maria Chiara Furlan, ${ }^{1}$ Francesca Carobbio, ${ }^{1}$ Alberto Cozzoli, \\ Tiziano Zanotelli, ${ }^{1}$ Claudio Simeone ${ }^{1}$
}

The presence of positive surgical margins (PSMs) after partial nephrectomy has been associated with an increased risk of disease recurrence, but conclusive evidence is lacking. The aim of this study is to examine the prognostic role of PSMs in a large retrospective cohort with long-term follow-up. PSMs were associated with disease recurrence, both local and distant, and decreased progression-free survival. Patients with PSMs should undergo a more intense follow-up.

Background: The role of positive surgical margins (PSMs) on the recurrence of renal cell carcinoma (RCC) after partial nephrectomy (PN) is debated, and available evidence lacks long-term data. The aim of this study was to evaluate the predictive role of PSMs on progression-free survival (PFS) in a large cohort followed for at least 5 years. Methods: This study was a retrospective analysis of a prospectively compiled single-institution database collecting complete information on more than 2700 patients who had undergone surgery for renal tumor. The data of all the patients submitted to PN for RCC and with least 5 years follow-up were extracted. Surgical specimens were examined at the time of surgery only by 2 expert uro-pathologists. A PSM was defined as the presence of cancer cells at the inked surface of the specimen. The role of PSMs on survival was estimated by Cox regression models adjusted for influent covariates. Results: A total of 459 patients fulfilled the inclusion criteria and were evaluated. PSMs were observed in 27 (5.9\%) cases. No differences in preoperative and pathologic data were found comparing patients with and without PSMs. At a median follow-up of 96 months (interquartile range, 74-131 months), a clinically evident relapse of RCC was diagnosed in $36(7.8 \%)$ patients at a median interval of 36 months from PN. Among these, 6 had a PSM for an incidence of relapse of $22.2 \%$ in the PSM group, whereas 30 had negative margins, for an incidence of $6.9 \%$ $(P=.013)$. The sites of relapse were distant organs in 18 cases, and the kidney underwent PN in 21. The patients with PSMs showed a borderline significantly higher incidence of distant metastasis $(11.1 \%$ vs. $3.5 \% ; P=.071)$ and a significantly higher incidence of renal relapses (14.8\% vs. 3.9\%; $P=.029)$. Multivariable Cox models confirmed that the presence of PSMs was an independent predictor of PFS (odds ratio, 3.127; $P=.013$ ). Conclusions: PSMs are an independent predictor of PFS in patients who underwent PN for RCC, owing to a higher incidence of distant and local relapses. Surveillance in presence of PSMs should be intensified and extended for a long time.

Clinical Genitourinary Cancer, Vol. 17, No. 1, e26-31 @ 2018 Elsevier Inc. All rights reserved. Keywords: Partial nephrectomy, Predictors, Prognosis, Recurrence, Renal Cell Carcinoma

R.T. and A.A. contributed equally to this work as first authors.

${ }^{1}$ Unit of Urology

${ }^{2}$ Unit of Pathology, Spedali Civili Hospital, University of Brescia, Brescia, Italy
Submitted: May 21, 2018; Revised: Aug 12, 2018; Accepted: Aug 14, 2018; Epub: Aug 23, 2018

Address for correspondence: Riccardo Tellini, MD, UO Urologia, ASST Spedali Civil di Brescia, Piazzale Spedali Civili 1, 25100, Brescia, Italy E-mail contact: riccatello@gmail.com 


\section{Introduction}

Renal cell carcinoma (RCC) represents $2 \%$ to $3 \%$ of solid tumors, and its incidence is increasing, especially in young patients. ${ }^{1}$ According to international guidelines, ${ }^{2,3}$ partial nephrectomy (PN) represents the standard of care for organ-confined RCC because it offers excellent oncologic results ${ }^{4}$ and better preservation of renal function $^{5}$ that could reduce cardiovascular and probably also cancerspecific mortality. ${ }^{6-8}$

Urologists are fairly concerned of the risk of positive surgical margins (PSMs) because this could jeopardize patients' survival, as happens for most oncologic surgeries. ${ }^{9,10}$ Thus, avoiding a PSM is paramount, and this goal has been included among all the composite tools designed to objectivize the outcomes of PN, like the Trifecta or the Margin Ischemia Complications score. ${ }^{11,12}$ However, the role of PSMs on the risk of recurrence of RCC is still under debate because the available evidence is made by retrospective studies with conflicting results. Several authors reported that a PSM could be relevant on prognosis only for RCC with pathologic aggressive features, ${ }^{13}$ but the majority undermined the potential negative role of PSMs. ${ }^{14}$ Nevertheless, almost all these studies provided a relatively short follow-up time that could have masked the prognostic role of PSMs, especially in case of the more indolent cancers for which relapses could occur at a very long distance. $^{15}$

The aim of this study is to evaluate the oncologic impact of PSMs in a large single center cohort of patients who underwent PN followed for at least 5 years.

\section{Materials and Methods}

This study was a retrospective review of a prospectively maintained institutional database including all clinical, surgical, pathologic, and follow-up data of more than 2700 consecutive patients who underwent surgery for a renal tumor since 1983. The indication of radical nephrectomy versus PN followed international guidelines contemporary to the date of surgery. Operations were performed through open retroperitoneal, laparoscopic, or robotassisted approaches, depending on the period of the study and surgeon's preference. After resection, at the discretion of the surgeon, punch biopsies of the tumor bed were taken and sent for frozen sections to assess the presence of residual disease and consistently proceed to wider resection or nephrectomy. According to institutional policy, the resection strategy for $\mathrm{PN}$ intended to leave a thin layer of healthy parenchyma around the tumor, by performing an enucleo-resection. Across the period of the study, 2 dedicated expert uro-pathologists (R.T. and S.F.) examined surgical specimens and defined PSM as the presence of cancer cells at the inked surface. RCC subtyping was updated to contemporary World Health Organization classification of renal tumors, ${ }^{16}$ nuclear grading was attributed according to Fuhrman criteria, ${ }^{17}$ and pathologic staging was assigned according to the Union for International Cancer Control TNM staging, 2017 edition. ${ }^{18}$

Patients underwent periodical follow-up controls at a dedicated outpatient office according to a schedule tailored on staging and grading, as previously reported. ${ }^{19}$ Briefly, a computed tomography scan was required at 4 months and then alternated with abdomen ultrasound plus chest $\mathrm{x}$-rays every 6 months for 2 years, and then yearly for an indefinite time. Recurrence was defined by the clinical evidence of lesions with features compatible with relapsing RCC, eventually confirmed at pathologic examination by biopsy or resection. A local recurrence was intended as the presence of a new tumor that arose from the previous site of PN identified by computed tomography by the hyper-dense plugs or clips used for the renorrhaphy. Both local recurrences and distant metastasis were generally candidates for surgical treatment whenever deemed as technically resectable, otherwise medical therapy was indicated.

All patients who underwent PN for nonmetastatic RCC with at least 5 years of follow-up were included in the present analysis. The prognostic role of the presence of PSMa at final pathology was investigated, accounting for other predictors of progression-free survival (PFS).

\section{Statistical Analysis}

Continuous variables were reported as median and interquartile range (IQR), and categorical variables as number and proportions. The Student $t$ test or Mann-Whitney $U$ test were used to compare continuous variables, and the Pearson $\chi^{2}$ test was used for categorical variables. The endpoint of the study was PFS, defined as the time between PN and the first event of a clinically documented recurrence or death owing to RCC, censoring the cases with recurrence. The status of patients who disregarded controls was updated by consultation of hospital or administrative online registries or phone calls. Univariate Cox models estimated the association between predictors, among which PSM was one, and PFS; multivariable models assessed the association between PSMs and PFS, accounting for other influent covariates selected by univariable analysis. Differences were considered statistically significant with $P \leq .05$, and all $P$ values were 2 -sided. Analyses were performed using SPSS (IBM Corp, IBM SPSS Statistics for Windows, Version 20.0, Armonk, NY).

\section{Results}

Over 1055 PNs for a renal tumor were performed after 1983; the data of 459 patients with final pathology consistent with RCC and a minimum follow-up of 5 years were reviewed. Across the long time span of the study, the majority of patients were operated on during the more recent years, as the cumulative incidence of $50 \%$ of cases was reached in 2004. A PSM was found in 27 patients (prevalence of PSMs, 5.9\%), with a higher rate in more recent cases (year of surgery 1983-2004 vs. 2005-2014, prevalence of PSM 3.8\% vs. $8.1 \% ; P=.072$ ). No differences were found in preoperative and pathologic features according to the status of surgical margins (Table 1). The cohort was followed for a median time of 96 months (IQR, 74-131 months) from PN to death or last control. Overall, 39 events of progression were diagnosed in $36(7.8 \%)$ patients, of whom 6 had a PSM (22\% of 27 with PSMs) and 30 a negative margin $(6.9 \%$ of 432 with negative margins) $(P=.013)$. The relapse occurred at distant sites in 18 patients and in the kidney that previously underwent $\mathrm{PN}$ in 21 . The incidence of distant metastasis in patients with PSMs was higher, with borderline statistical significance $(3 / 27$ vs. $15 / 432$, or $11.1 \%$ vs. $3.5 \% ; P=.071)$, whereas the higher incidence of local relapse in the patients with 


\section{Positive Surgical Margins After Nephron-Sparing Surgery}

Table 1 Preoperative and Pathologic Features of Patients

\begin{tabular}{|c|c|c|c|}
\hline & $\begin{array}{c}\text { PSMs } \\
27 \text { Patients }(5.9 \%) \text {, n (\%) }\end{array}$ & $\begin{array}{c}\text { No PSMs } \\
432 \text { Patients }(94.1 \%) \text {, n (\%) }\end{array}$ & $P$ Value \\
\hline Gender & & & .982 \\
\hline Male & $18(66.7)$ & $310(66.9)$ & \\
\hline Female & 9 (33.3) & $153(33.1)$ & \\
\hline Mean age at surgery, y (SD) & $62.2( \pm 10.2)$ & $60.7( \pm 12.7)$ & .719 \\
\hline Mean clinical diameter, cm (SD) & $3.2( \pm 1.3)$ & $3.1( \pm 1.3)$ & .832 \\
\hline pT stage & & & .221 \\
\hline pT1a & $21(77.7)$ & $349(80.8)$ & \\
\hline pT1b & $3(11.1)$ & $61(14.1)$ & \\
\hline pT2 & $1(3.8)$ & $6(1.4)$ & \\
\hline рТ3a & $2(7.4)$ & $16(3.7)$ & \\
\hline RCC subtype & & & .361 \\
\hline Clear cell & $18(66.7)$ & $319(73.8)$ & \\
\hline Papillary & $5(18.5)$ & $74(17.1)$ & \\
\hline Chromophobe & $3(11.1)$ & $28(6.5)$ & \\
\hline Others & $1(3.7)$ & $11(2.6)$ & \\
\hline
\end{tabular}

Abbreviations: PSMs $=$ Positive surgical margins; $\mathrm{RCC}=$ renal cell carcinoma.

PSM reached statistical significance ( $4 / 27$ vs. $17 / 432$, or $14.8 \%$ vs. $3.9 \% ; P=.029)$. The median time to distant metastasis was 46 months (IQR, 11-80 months) in the PSM group and 39 months (IQR, 15-50 months) in other patients $(P=.147)$, whereas the median time to local recurrence was, respectively, 43 months (IQR, 17-68 months) and 56 months (IQR, 26-96 months) $(P=.56)$. Multivariable models showed that tumor diameter (odds ratio [OR], 1.269; $P<.001$ ), pathologic staging and, in particular, pT3a stage (OR, 5.734; $P<.001$ ), high Fuhrman grading (OR, 5.346; $P=.006)$, and PSMs (OR, 3.127; $P=.013$ ) were independently predictive of PFS (Table 2).

At the diagnosis of relapse, 23 patients underwent surgical treatment (16 radical nephrectomies, 7 metastasectomies), 7 patients had medical therapy, and 6 patients received supportive care. Among the patients who underwent surgery for the relapse, 10 achieved a long control of the disease, remaining free from RCC at a median interval of 21 months (IQR, 14-70 months) from recurrence (see Supplemental Table 1 in the online version).

\section{Discussion}

The present study provided an insight on issue of the prognostic role of PSMs after PN, relying on a cohort with the longest followup reported until now (median time, 8 years). Moreover, the population has homogeneous features in terms of surgical management and, most of all, pathologic assessments, because all patients were treated at 1 institution with only 2 expert uropathologists involved. Within our experience, a PSM was detected approximately in $6 \%$ of PNs for RCC; that is one-half of the range reported by the literature $(0 \%-15 \%){ }^{20}$

Table 2 Univariate and Multivariable Cox Regression Models for Progression-free Survival After Partial Nephrectomy

\begin{tabular}{|c|c|c|c|c|}
\hline \multirow[b]{2}{*}{ Variable } & \multicolumn{2}{|c|}{ Univariate } & \multicolumn{2}{|c|}{ Multivariable } \\
\hline & OR (95\% Cl) & $\boldsymbol{P}$ & OR (95\% Cl) & $\boldsymbol{P}$ \\
\hline Tumor diameter, $\mathrm{cm}$ & $1.340(1.145-1.567)$ & $<.001$ & $1.269(1.065-1.511)$ & $<.001$ \\
\hline Pathologic stage & & .001 & & $<.001$ \\
\hline pT1a & Referent & & Referent & \\
\hline pT1b & $0.935(0.325-2.686)$ & .9 & $0.680(0.265-1.742)$ & .42 \\
\hline pT2 & $2.114(0.286-15.621)$ & .47 & $1.922(0.347-13.129)$ & .19 \\
\hline рТ3а & $6.213(2.541-15.193)$ & $<.001$ & $5.734(2.356-13.958)$ & $<.001$ \\
\hline High grading & $5.399(1.653-17.632)$ & .005 & $5.346(1.629-17.550)$ & .006 \\
\hline Histologic subtype $^{a}$ & $0.842(0.557-1.273)$ & .41 & & \\
\hline Necrosis & $1.042(0.249-4.360)$ & .955 & & \\
\hline Microvascular invasion & $2.005(0.274-14.666)$ & .493 & & \\
\hline PSMs & 3.608 (1.499-8.683) & .004 & 3.127 (1.272-7.688) & .013 \\
\hline
\end{tabular}

Abbreviations: $\mathrm{Cl}=$ Confidence interval; $\mathrm{OR}=$ odds ratio; $\mathrm{PSMs}=$ positive surgical margins.

${ }^{\mathrm{a} C l e a r-c e l l}$ renal carcinoma versus other histologic subtypes. 
The first finding is that the clinical and pathologic features of the cases with and without PSMs were overlapping, indicating that this phenomenon is poorly predictable on the basis of the features of the patient or the tumor, but probably relies on drawbacks of surgical dissection. Splitting the cohort on 2 periods with the same number of procedures, before and after 2005, an increase in the incidence of PSMs was observed, reasonably because of the expansion of the indications for PN. ${ }^{21}$ Our data showed an increased risk of recurrence of RCC in the presence of PSMs, both at distant sites and, even more, as relapses in the operated kidney. This involves that the follow-up schedule after PSMs should include both abdominal and extra-abdominal — definitely chest-imaging examinations. In addition, the higher number of local recurrences after PSMs confirms that a new tumor could grow when malignant cells are left on the resection bed, as recently shown by a study that reviewed the pathologic findings at salvage nephrectomy for a relapse after PN. ${ }^{22}$ It must therefore be noted that any effort to achieve the complete removal of the tumor at the time of PN should be done. Of note, the median time of recurrence in our cohort was approximately 3 years, but occurred within a maximum of 8 years, suggesting that the follow-up in the case of PSMs might be prolonged. Finally, we found that in case of recurrences suitable for surgical treatment, a significant number of cases achieved long-term control of the disease: this corroborates the utility of controls after PN in order to allow an early diagnosis of recurrence.

The complete removal of the tumor is an obvious primary goal of oncologic surgery, but differently from other fields in which PSMs have a definite prognostic role and, in turn, specific management $^{23,24}$; only a little is known about the real harm related to PSMs after PN. International guidelines often neglect this issue ${ }^{3}$ or broadly suggest an "intense surveillance," without providing further details. ${ }^{2}$ The results from existing studies on the prognostic role of PSMs are conflicting. Furthermore, considering that RCC, especially when poorly aggressive, as in a consistent number of the cases undergoing PN, could progress at a long distance, ${ }^{15}$ the majority of studies are based on a potentially insufficient follow-up time. ${ }^{20}$ Khalifeh et $\mathrm{al}^{25}$ in a large retrospective multicenter series of patients who underwent robot-assisted PN at a median time of 13 months, showed that PSMs were associated with a higher risk of local recurrence and metastasis with a hazard ratio of $18.4(P<.01)$ and $78.0(P=.02)$, respectively. Bansal et $\mathrm{al}^{26}{ }^{26}$ in a large population study with a median follow-up of 15 months, reported that PSMs were associated with a higher risk of progression. Shah et $\mathrm{al}^{13}$ reported on a multicentric cohort with a median follow-up time of 33 months and concluded that PSMs were predictive of recurrence-free survival, but only for high-risk tumors (high grading, pT3). Shum et $\mathrm{al}^{, 27}$ in a large matched comparison analysis based on the National Cancer Database, found a significant worse overall survival in the PSM group (hazard ratio, 1.393; $P=.001$ ).

On the other hand, the results from several other studies undermined the prognostic significance of PSMs. In a large multicenter study ${ }^{28}$ with a median follow-up time of 37 months, PSMs were associated with a higher risk of local recurrence but not with impaired cancer-specific survival at 5 years. Kang et $\mathrm{al}^{29}$ in a nationwide study on a homogenous cohort of pT1 clear cell RCC, largely low-grade, followed for a median time of 32 months, reported no differences in PFS.

As highlighted above, there is no consensus on the oncologic impact of surgical margins. It must be underlined that most studies are multicentric and therefore suffer from a significant bias related to the variability of margins assessment and lack of central review of specimens. Second, the longest follow-up times so far reported are very close to the median value of the latency we observed, having the advantage of a cohort followed for a significantly longer time: it could be hypothesized that a certain number of events of recurrence were missed without a prolonged control. Finally, multicentric studies cannot adequately report data on resection technique that could influence the risk of PSMs. Wang et $\mathrm{al}^{30}$ analyzed 117 tumors removed by simple enucleation or standard $\mathrm{PN}$ and found that the first had a higher rate of PSMs (17\% vs. $0 \%)$. Takagi et $\mathrm{al}^{31}$ instead reported that the 2 resection techniques lead to similar PSMs. Only with the diffuse application of a systematic nomenclature, as suggested by the Surface-Intermediate-Base score, ${ }^{32}$ a proper comparison could be done.

Our study has several limitations to be declared, starting from the retrospective design. The exclusion of the patients still without a minimal follow-up of 5 years may have led to unexpected selection biases. Even if only 2 expert uro-pathologists were involved, a slide review could have added some information, such as the extent of margin involvement in the case of PSMs. The utility of frozen sections to protect from PSMs could not have been estimated because this data was not separately recorded.

\section{Conclusion}

A PSM is an independent predictor of PFS in patients submitted to PN for RCC owing to a higher rate of local relapses of disease and distant metastasis. Until conclusive evidence is able to reveal the best management of patients with PSMs, a tailored intense and prolonged follow-up is mandatory to allow early diagnosis of relapse and its surgical resection.

\section{Clinical Practice Points}

- The role of surgical margin status after PN for RCC is still debated and no strong conclusions are available.

- In our study, with a long-term follow-up, we managed to demonstrate the negative prognostic role of PSMs in predicting disease recurrence.

- These findings suggest an intense follow-up in patients with PSMs after conservative surgery for kidney tumors in consideration of the increased risk of disease recurrence.

\section{Disclosure}

The authors have stated that they have no conflicts of interest.

\section{Supplemental Data}

Supplemental table accompanying this article can be found in the online version at https://doi.org/10.1016/j.clgc.2018.08.004. 


\section{Positive Surgical Margins After Nephron-Sparing Surgery}

\section{References}

1. King SC, Pollack LA, Li J, King JB, Master VA. Continued increase in incidence of renal cell carcinoma, especially in young patients and high grade disease: United States 2001 to 2010. J Urol 2014; 191:1665-70.

2. European Urology Association (EAU). Guidelines on Renal Cell Carcinoma, 2017 Update, Available at: https://uroweb.org/guideline/renal-cell-carcinoma. Accessed: April 24, 2018.

3. American Urological Association (AUA). Guidelines on Renal Mass and Localized Renal Cancer, 2017, Available at: http://www.auanet.org/guidelines/renal-massand-localized-renal-cancer-new-(2017). Accessed: April 24, 2018.

4. Van Poppel H, Da Pozzo L, Albrecht W, et al. A prospective, randomised EORTC intergroup phase 3 study comparing the oncologic outcome of elective nephronsparing surgery and radical nephrectomy for low-stage renal cell carcinoma. Eur Urol 2011; 59:543-52.

5. Scosyrev E, Messing EM, Sylvester R, Campbell S, Van Poppel H. Renal function after nephron-sparing surgery versus radical nephrectomy: results from EORTC randomized trial 30904. Eur Urol 2014; 65:372-7.

6. Capitanio U, Terrone C, Antonelli A, et al. Nephron-sparing techniques independently decrease the risk of cardiovascular events relative to radical nephrectomy in patients with a T1a-T1b renal mass and normal preoperative renal function. Eur Urol 2015; 67:683-9.

7. Antonelli A, Mari A, Longo N, et al, Collaborators. Role of clinical and surgical factors for the prediction of immediate, early and late functional result and its relatonship with cardivascular outcome after partial nephrectomy: results from the prospective multicenter RECORd 1 project. J Urol 2018; 199: 927-32.

8. Antonelli A, Minervini A, Sandri M, et al. Below safety limits, every unit of glomerular filtration rate counts: assessing the relationship between renal function and cancer-specific mortality in renal cell carcinoma. Eur Urol 2018. https://doi. org/10.1016/j.eururo.2018.07.029 [Epub ahead of print].

9. Jo JK, Hong SK, Byun SS, Zargar H, Autorino R, Lee SE. Positive surgical margin in robot-assisted radical prostatectomy: correlation with pathology findings and risk of biochemical recurrence. Minerva Urol Nefrol 2017; 69:493-500.

10. Hong X, Li T, Ling F, et al. Impact of surgical margin status on the outcome of bladder cancer treated by radical cystectomy: a meta-analysis. Oncotarget 2017; 8: 17258-69.

11. Buffi N, Lista G, Larcher A, et al. Margin, ischemia, and complications (MIC) score in partial nephrectomy: a new system for evaluating achievement of optimal outcomes in nephron-sparing surgery. Eur Urol 2012; 62:617-8.

12. Hung AJ, Cai J, Simmons MN, Gill IS. "Trifecta" in partial nephrectomy. J Urol 2013; 189:36-42.

13. Shah PH, Moreira DM, Okhunov Z, et al. Positive surgical margins increase risk of recurrence after partial nephrectomy for high risk renal tumors. J Urol 2016; 196:327-34.

14. Borghesi M, Brunocilla E, Schiavina R, Martorana G. Positive surgical margins after nephron-sparing surgery for renal cell carcinoma: incidence, clinical impact, and management. Clin Genitourin Cancer 2013; 11:5-9.

15. Antonelli A, Furlan M, Sodano M, et al. Features, risk factors and clinical outcome of "very late" recurrences after surgery for localized renal carcinoma: a retrospective evaluation of a cohort with a minimum of 10 years of follow up. Int J Urol 2016; 23:36-40.
16. Moch H, Cubilla AL, Humphrey PA, Reuter VE, Ulbright TM. The 2016 WHO classification of tumours of the urinary system and male genital organs-part A: renal, penile, and testicular tumours. Eur Urol 2016; 70:93-105.

17. Fuhrman SA, Lasky LC, Limas C. Prognostic significance of morphologic parameters in renal cell carcinoma. Am J Surg Pathol 1982; 6:655-63.

18. Brierley JD, Gospodarowicz MK, Wittekind C. TNM Classification of Malignant Tumours. 8th Edition. Hoboken, NJ: Wiley-Blackwell; 2016.

19. Antonelli A, Cozzoli A, Zani D, et al. The follow-up management of nonmetastatic renal cell carcinoma: definition of a surveillance protocol. BJU Int 2007; 99:296-300.

20. Minervini A, Campi R, Sessa F, et al. Positive surgical margins and local recurrence after simple enucleation and standard partial nephrectomy for malignant renal tumors: systematic review of the literature and meta-analysis of prevalence. Minerva Urol Nefrol 2017; 69:523-38.

21. Antonelli A, Cozzoli A, Nicolai M, et al. Nephron-sparing surgery versus radical nephrectomy in the treatment of intracapsular renal cell carcinoma up to $7 \mathrm{~cm}$. Eur Urol 2008; 53:803-9.

22. Antonelli A, Furlan M, Tardanico R, et al. Features of ipsilateral renal recurrences after partial nephrectomy: a proposal of a pathogenetic classification. Clin Genitourin Cancer 2017; 15:540-7.

23. Mari A, Campi R, Tellini R, et al. Patterns and predictors of recurrence after open radical cystectomy for bladder cancer: a comprehensive review of the literature. World J Urol 2018; 36:157-70.

24. Yossepowitch O, Bjartell A, Eastham JA, et al. Positive surgical margins in radical prostatectomy: outlining the problem and its long-term consequences. Eur Urol 2009; 55:87-99.

25. Khalifeh A, Kaouk JH, Bhayani S, et al. Positive surgical margins in robot-assisted partial nephrectomy: a multi-institutional analysis of oncologic outcomes (leave no tumor behind). J Urol 2013; 190:1674-9.

26. Bansal RK, Tanguay S, Finelli A, et al. Positive surgical margins during partial nephrectomy for renal cell carcinoma: results from Canadian Kidney Cancer information system (CKCis) collaborative. Can Urol Assoc J 2017; 11:182-7.

27. Shum CF, Bahler CD, Sundaram CP. Impact of positive surgical margins on overall survival after partial nephrectomy-a matched comparison based on the National Cancer Database. Urol Oncol 2018; 36:90.e15-21.

28. Bensalah K, Pantuck AJ, Rioux-Leclercq N, et al. Positive surgical margin appears to have negligible impact on survival of renal cell carcinomas treated by nephronsparing surgery. Eur Urol 2010; 57:466-71.

29. Kang HW, Lee SK, Kim WT, et al. Surgical margin does not influence recurrence rate in pT1 clear cell renal cell carcinoma after partial nephrectomy: a multicenter study. J Surg Oncol 2016; 114:70-4.

30. Wang L, Hughes I, Snarskis C, et al. Tumor enucleation specimens of small renal tumors more frequently have a positive surgical margin than partial nephrectomy specimens, but this is not associated with local tumor recurrence. Virchows Arch 2017; 470:55-61.

31. Takagi T, Kondo T, Tachibana H, et al. Comparison of surgical outcomes between resection and enucleation in robot-assisted laparoscopic partial nephrectomy for renal tumors according to the surface-intermediate-base margin score: a propensity score-matched study. J Endourol 2017; 31:756-61.

32. Minervini A, Carini M, Uzzo RG, Campi R, Smaldone MC, Kutikov A. Standardized reporting of resection technique during nephron-sparing surgery: the surface-intermediate-base margin score. Eur Urol 2014; 66:803-5. 


\section{Supplemental Table 1 Features of Patients With Disease Recurrence After PN for RCC}

\begin{tabular}{|c|c|c|c|c|}
\hline Patient No. & Site of Recurrence & $\begin{array}{c}\text { Time to } \\
\text { Recurrence, mo }\end{array}$ & Primary Tumor Features & Treatment of Recurrence \\
\hline 1 & Operated kidney + surgical incision & 11 & ccRCC G3 pT3a & Surgery (RN) \\
\hline 2 & Operated kidney & 23 & ccRCC G3 pT1a & Surgery (RN) \\
\hline 3 & Operated kidney & 40 & ccRCC G4 pT1a & Surgery (RN) \\
\hline 4 & Operated kidney & 96 & pRCC pT1b & Surgery (RN) \\
\hline 5 & Operated kidney & 18 & ccRCC G4 pT3a & Surgery (RN) \\
\hline 6 & Operated kidney & 13 & ccRCC G3 pT1a & Surgery (RN) \\
\hline 7 & Operated kidney + operated renal fossa & 37 & ccRCC G4 (sarcomatoid features) pТЗa & Best supportive care \\
\hline 8 & Operated kidney & 34 & ccRCC G2 pT1a & Surgery (RN) \\
\hline 9 & Operated kidney & 42 & ccRCC G3 pT1a & Surgery (RN) \\
\hline 10 & Operated kidney & 49 & ccRCC G3 pT1a & Surgery (RN) \\
\hline 11 & Operated kidney + operated renal fossa & 51 & ccRCC G3-4 pT1b & Surgery (RN) \\
\hline 12 & Operated kidney & 34 & pRCC pT1a & Surgery (RN) \\
\hline 13 & Operated kidney + Lung & 50 & ccRCC G3 pT1b & Medical therapy \\
\hline 14 & Operated kidney & 74 & ccRCC G2 pT1a & Surgery (RN) \\
\hline 15 & Operated kidney & 9 & ccRCC G3 pT1b & Medical therapy \\
\hline 16 & Operated kidney & 101 & chRCC pT1a & Surgery (RN) \\
\hline 17 & Operated kidney & 50 & ccRCC G3 pT1a & Surgery (partial nephrectomy) \\
\hline 18 & Operated kidney & 96 & ccRCC G2 pT1b & Surgery (RN) \\
\hline 19 & Operated kidney & 52 & pRCC pT1a & Surgery (RN) \\
\hline 20 & Operated kidney & 50 & ccRCC G2 pT1a & Surgery (partial nephrectomy) \\
\hline 21 & Operated kidney & 116 & ccRCC G3 pT1a & Surgery (RN) \\
\hline 22 & Pancreas & 45 & ccRCC G3 pT1a & Surgery (metastasectomy) \\
\hline 23 & Lung & 50 & pRCC & Surgery (metastasectomy) \\
\hline 24 & Liver & 29 & ccRCC G3 pT1b & Surgery (metastasectomy) \\
\hline 25 & Lung & 48 & ccRCC G2 pT1a & Medical therapy \\
\hline 26 & Lung & 32 & ccRCC G3/4 pT1b & Medical therapy \\
\hline 27 & Bones & 12 & ccRCC G4 pT3a & Best supportive care \\
\hline 28 & Lung & 65 & ccRCC G3 pT1a & Surgery (metastasectomy) \\
\hline 29 & Lung & 83 & $\mathrm{pRCC}$ & Medical therapy \\
\hline 30 & Bones & 36 & ccRCC G2 pT1a & Medical therapy \\
\hline 31 & Lung & 78 & ccRCC G3 pT1b & Medical therapy \\
\hline 32 & Brain & 8 & ccRCC G3 pT1a & Medical therapy \\
\hline 33 & Contralateral adrenal gland & 101 & ccRCC G2 pT1a & Surgery (metastasectomy) \\
\hline 34 & Pancreas & 35 & ccRCC G2 pT1a & Surgery (metastasectomy) \\
\hline 35 & Bones & 10 & pRCC G3 pT1b & Best supportive care \\
\hline 36 & Lung & 47 & ccRCC G2 pT1a & Surgery (metastasectomy) \\
\hline
\end{tabular}

Abbreviations: $\mathrm{cCRCC}=$ Clear-cell renal cell carcinoma; $\mathrm{PRCC}=$ papillary renal cell carcinoma; PSM = positive surgical margin; RCC = renal cell carcinoma; $\mathrm{RN}=$ radical nephrectomy. 